\title{
Radiation transport of heliospheric Lyman- $\alpha$ from combined Cassini and Voyager data sets
}

\author{
W. Pryor $^{1,2}$, P. Gangopadhyay ${ }^{3}$, B. Sandel ${ }^{4}$, T. Forrester ${ }^{4}$, E. Quemerais ${ }^{5}$, E. Möbius ${ }^{6}$, L. Esposito ${ }^{7}$, I. Stewart ${ }^{7}$, \\ W. McClintock ${ }^{7}$, A. Jouchoux ${ }^{7}$, J. Colwell ${ }^{8}$, V. Izmodenov ${ }^{9,10}$, Y. Malama ${ }^{10}$, K. Tobiska ${ }^{2}$, D. Shemansky ${ }^{2}$, J. Ajello ${ }^{11}$, \\ C. Hansen ${ }^{11}$, and M. Bzowski ${ }^{12}$ \\ ${ }^{1}$ Central Arizona College, 8470 N. Overfield Rd., Coolidge, AZ 85228, USA \\ e-mail: wayne.pryor@centralaz.edu \\ 2 Space Environment Technologies, 1676 Palisades Dr., Pacific Palisades, CA 90272, USA \\ 3 Department of Physics, Astronomy and Space Sciences Center, University of Southern California, Los Angeles, CA, 90089, USA \\ ${ }^{4}$ Lunar and Planetary Laboratory, University of Arizona, 1541 E University Blvd, Tucson, AZ 85721-0077, USA \\ 5 Service d'Aéronomie, BP 3, 91371 Verrières-le-Buisson, France \\ 6 Dept. of Physics, University of New Hampshire, Durham, NH 03824, USA \\ ${ }^{7}$ Laboratory for Atmospheric and Space Physics, University of Colorado, 1234 Innovation Dr., Boulder, CO 80303, USA \\ 8 Dept. of Physics, University of Central Florida, 4000 Central Florida Boulevard, Building 12 Room 310, Orlando, \\ FL 32816-2385, USA \\ 9 Lomonosov Moscow State University, Department of Aeromechanics and Gas Dynamics, School of Mechanics and Mathematics, \\ Moscow 119899, Russia \\ 10 Space Research Institute (IKI) and Institute for Problems in Mechanics, Russian Academy of Sciences, Prospect Vernadskogo 101, \\ Moscow 117526, Russia \\ 11 Jet Propulsion Laboratory, 4800 Oak Grove Dr., Pasadena, CA 91109, USA \\ 12 Space Research Centre, Polish Academy of Sciences, Bartycka 18A, 00-716 Warsaw, Poland
}

Received 17 October 2007 / Accepted 5 June 2008

ABSTRACT

\begin{abstract}
Aims. Heliospheric neutral hydrogen scatters solar Lyman- $\alpha$ radiation from the Sun with "27-day" intensity modulations observed near Earth due to the Sun's rotation combined with Earth's orbital motion. These modulations are increasingly damped in amplitude at larger distances from the Sun due to multiple scattering in the heliosphere, providing a diagnostic of the interplanetary neutral hydrogen density independent of instrument calibration.

Methods. This paper presents Cassini data from 2003-2004 obtained downwind near Saturn at $~ 10$ AU that at times show undamped "27-day" waves in good agreement with the single-scattering models of Pryor et al. (1992, ApJ, 394, 363). Simultaneous Voyager 1 data from 2003-2004 obtained upwind at a distance of 88.8-92.6 AU from the Sun show waves damped by a factor of $\sim 0.21$. The observed degree of damping is interpreted in terms of Monte Carlo multiple-scattering calculations (e.g., Keller et al. 1981, A\&A, 102, 415) applied to two heliospheric hydrogen two-shock density distributions (discussed in Gangopadhyay et al. 2006, ApJ, 637, 786) calculated in the frame of the Baranov-Malama model of the solar wind interaction with the two-component (neutral hydrogen and plasma) interstellar wind (Baranov \& Malama 1993, J. Geophys. Res., 98, 15157; Izmodenov et al. 2001, J. Geophys. Res., 106, 10681; Baranov \& Izmodenov 2006, Fluid Dyn., 41, 689).

Results. We conclude that multiple scattering is definitely occurring in the outer heliosphere. Both models compare favorably to the data, using heliospheric neutral $\mathrm{H}$ densities at the termination shock of $0.085 \mathrm{~cm}^{-3}$ and $0.095 \mathrm{~cm}^{-3}$. This work generally agrees with earlier discussions of Voyager data in Quemerais et al. (1996, ApJ, 463, 349) showing the importance of multiple scattering but is based on Voyager data obtained at larger distances from the Sun (with larger damping) simultaneously with Cassini data obtained closer to the Sun.
\end{abstract}

Key words. ISM: atoms - interplanetary medium - Sun: UV radiation

\section{Introduction}

Interplanetary Lyman- $\alpha$ radiation, first detected by the Venus probe Zond 1 (Kurt 1967), is the brightest ultraviolet emission from interplanetary gas. Subsequent Lyman- $\alpha$ observations from the Orbiting Geophysical Observatory (OGO-5) showing a bright upwind and dimmer downwind direction suggested that the gas was interstellar in origin and flowing through the solar system (Bertaux \& Blamont 1971; Thomas \& Krassa 1971). The first spectrum of the interplanetary glow from
Copernicus showed it has the same characteristics (velocity and density) as the nearby interstellar gas in the upwind direction (Adams \& Frisch 1977). Early research reviewed in Thomas 1978 concludes that interplanetary Lyman- $\alpha$ is produced by resonance scattering of solar Lyman- $\alpha$ by interstellar hydrogen gas approaching the Sun from the upwind direction. The best determination of the upwind direction was made using the Ulysses GAS experiment (Witte et al. 1996) to measure the neutral helium flow. In J2000 coordinates, helium approaches the Sun at $26.3 \pm 0.4 \mathrm{~km} \mathrm{~s}^{-1}$ from ecliptic longitude $255.4 \pm 0.5$ degrees 
and ecliptic latitude $5.1 \pm 0.2$ degrees (Witte 2004, 2007, private communication). Hydrogen loss processes near the Sun (primarily charge-exchange with solar wind protons, and a smaller contribution from solar EUV photoionization) lead to a cavity depleted in slow neutral $\mathrm{H}$ that can scatter Lyman- $\alpha$.

The heliospheric Lyman- $\alpha$ intensity seen in any direction varies as the Sun rotates, because the Sun's ultraviolet emissions are enhanced in localized active regions, generally at low solar latitudes. This modulation in the heliospheric Lyman- $\alpha$ intensity can be estimated from a hydrogen hot model that calculates hydrogen densities (e.g., Thomas 1978) coupled to a single-scattering radiative transfer calculation that integrates model emission rates along a given line of sight that can then be compared to data. The emissions in each direction from the Sun are estimated using solar Lyman- $\alpha$ values measured by spacecraft near Earth such as UARS (Upper Atmosphere Research Satellite) SOLSTICE (Solar-Stellar Irradiance Comparison Experiment) or TIMED (Thermosphere Ionosphere Mesophere Energetics and Dynamics) SEE (Solar EUV Experiment). Hot models neglect outer heliospheric effects on the hydrogen population, but are still frequently used to describe the hydrogen population inside the termination shock. As first demonstrated by Shemansky et al. (1984), the Lyman- $\alpha$ modulations seen in heliospheric data are reduced in amplitude in the outer heliosphere compared to the single-scattering models, providing evidence that multiple scattering is significant in forming the observed emission. Multiple scattering acts to reduce the flux differences observed in the heliosphere by increasing the range of angles over which a localized solar bright spot illuminates the heliosphere (Quemerais et al. 1996). Light travel-time effects from multiple scattering could also act to reduce the 27-day brightness modulations, but are a minor effect, since most of the scatterings of interest occur within 1 light-day of the Sun (1 AU corresponds to 8 light-minutes).

In this paper, we compare recent Voyager and Cassini data sets to a single-scattering model, demonstrate the presence of damping in the Voyager data, and then assess the resulting damping factors (modulation amplitude/near-sun modulation amplitude) in terms of multiple scattering models in order to derive an estimate of the interplanetary $\mathrm{H}$ density at large distances from the Sun, but inside the $\mathrm{H}$ wall outside the recently detected (Stone et al. 2005) termination shock seen at 94 AU from the Sun. The termination shock (TS) is formed by the interaction of the solar wind with the plasma component of the interstellar medium together with the heliopause (HP) and possibly the bow shock (BS) (see Fig. 1). The atoms of interstellar hydrogen penetrate into the plasma interaction region (the region between the BS and TS that is often called the heliospheric interface) due to their large mean free path. Inside the heliospheric interface the $\mathrm{H}$ atoms charge-exchange with protons and are decelerated and heated. As the result of the charge exchange new (socalled secondary) interstellar atoms are created. These secondary atoms reflect properties of the interstellar protons in the region between the HP and BS, i.e. they are hotter and slower as compared with original (or primary) interstellar atoms. Note, that these are the secondary interstellar atoms that collectively with the primaries make up the hydrogen wall around the heliopause. This hydrogen wall was first predicted theoretically by Baranov et al. (1991) and then observed by Linsky \& Wood (1996) in absorption on the line-of-sight towards the star $\alpha$ Centauri. The heliospheric hydrogen wall has now been observed in many directions (see, e.g. recent papers by Wood et al. 2007a,b, and references therein).

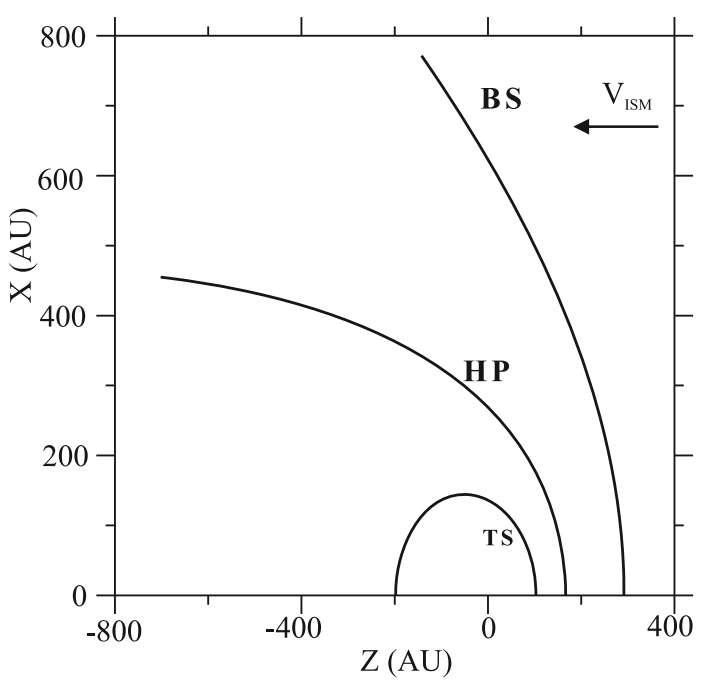

Fig. 1. Illustrates how a flow of interstellar neutrals and plasma at velocity $V_{\text {ISM }}$ from the upwind direction interacting with the solar wind flowing radially outwards from the Sun (located at $Z=0, X=0$ ) sets up a bow shock (BS), heliopause (HP), and solar wind termination shock (TS) in a Baranov-Malama type model.

Since the interstellar hydrogen is processed in the heliospheric interface before it penetrates the termination shock, our estimates of the hydrogen density will not refer to the local interstellar medium value, but rather the "processed value" after charge-exchange filtration through the outer heliospheric shock structures has reduced the interstellar neutral hydrogen density to a lower density level near the termination shock. These estimates will be compared to other $\mathrm{H}$ density estimates near the termination shock from interstellar absorption measurements, from hydrogen pickup ion measurements and from solar wind slowdown measurements described in companion papers in this issue (Slavin \& Frisch 2008; Bzowski et al. 2008; Richardson et al. 2008).

\section{Observations}

We will mention interplanetary Lyman- $\alpha$ data from five spacecraft instruments: the Pioneer UV photometers (Judge \& Carlson 1974; Carlson \& Judge 1974) mounted on Pioneer 10, leaving the solar system in the downwind direction, and on Pioneer 11, leaving upwind; the Voyager Ultraviolet Spectrometers (UVS, Broadfoot et al. 1977), mounted on the Voyager 1 and 2 spacecraft and now leaving the solar system in the upwind direction; and the Cassini Ultraviolet Imaging Spectrograph (UVIS, Esposito et al. 2004) on the Cassini orbiter mission to Saturn, downwind at Saturn arrival in 2004. Specific examples to be discussed are taken from 3 different solar cycles. Periods near solar maximum are best because at that time large active regions on the Sun are most likely to be present, creating 27-day waves of significant amplitude.

The first example, presented by Shemansky et al. (1984), was Voyager 2 and Pioneer 10 interplanetary Lyman- $\alpha$ modulation data from 1982 used to infer hot model hydrogen density at large distances from the sun. Voyager 2 interplanetary data obtained at $\sim 12 \mathrm{AU}$ out from the Sun in 1982 show modulations almost as large as the modulations in the Solar Mesosphere Explorer (SME) solar Lyman- $\alpha$ variation. Shemansky et al. (1984) interpreted the Voyager data as indicating the outer heliospheric neutral hydrogen density $=0.16-0.17 \mathrm{~cm}^{-3}$ based on multiple 


\section{CASSINI INTERPLANETARY TRAJECTORY}

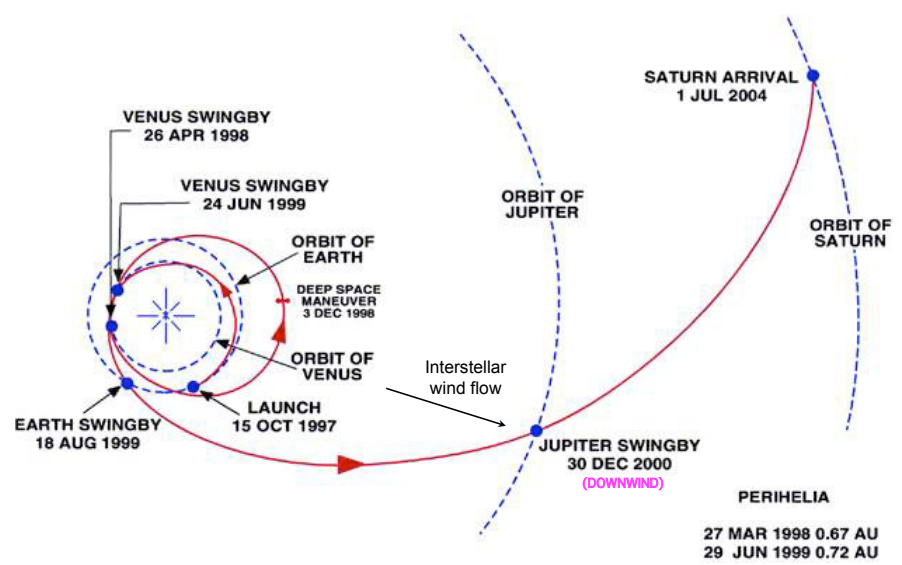

Fig. 2. The Cassini spacecraft's trajectory to Saturn placed it downwind with respect to the interstellar hydrogen flow in late 2000 during the Jupiter swingby. The view is looking from ecliptic north down onto the ecliptic plane. Downwind is indicated in magenta. The Cassini spacecraft was somewhat more sidewind in 2003-2004. Voyager 1 was upwind during this period.

scattering calculations for a hot hydrogen model presented in Keller et al. (1981). Pioneer 10 data from 30 AU out from the Sun from the same period in 1982 show much smaller modulations, that Shemansky et al. (1984) interpreted as resulting from multiple scattering by the outer heliospheric hydrogen with a density $=0.11-0.12 \mathrm{~cm}^{-3}$.

The second example, presented by Quemerais et al. (1996), used Voyager data from 1981-1993. At the end of that period, they found that the estimated solar Lyman- $\alpha$ line-center flux modulation is damped by a factor of 0.4 (or smaller) in the Voyager (1 and 2) data, when the spacecraft were at distances of 56 and $44 \mathrm{AU}$ respectively. They interpreted their data with a hot model for the hydrogen distribution and a Monte Carlo calculation for photon scattering, and concluded that the observed degree of damping was consistent with a hydrogen density of $0.15 \pm 0.10 \mathrm{~cm}^{-3}$.

The third example, previously unpublished, comes from examination of recent Voyager 1 UVS upwind data (looking generally upwind, with instrument pointings near ecliptic longitude 263 degrees, ecliptic latitude 20 degrees) from 2003-2004 obtained from 88.8-92.6 AU from the Sun and comparisons with simultaneous measurements of the upwind hemisphere from the downwind Cassini UVIS as it approached Saturn. Figure 2 shows the Cassini trajectory, while Fig. 3 shows a high signalto-noise ratio UVIS Lyman- $\alpha$ spectrum. The UVIS data spatial and temporal variations are in reasonable agreement with an optically thin model to be discussed below. The "27-day" waves due to solar rotation in the Voyager data and the optically thin model also generally agree in phase and shape, but the waves in the data are damped by a factor of about 0.21 compared to model values produced in the optically thin model.

\section{Optically thin model}

Hot models for hydrogen (e.g., Thomas 1978) begin with initial thermodynamic parameters for the neutral hydrogen at large distances from the sun (usually assumed to be near the termination shock). These key parameters are the neutral hydrogen density, temperature, and velocity. For density we try $0.085 \mathrm{~cm}^{-3}$, to match the termination shock value from a Baranov-Malama

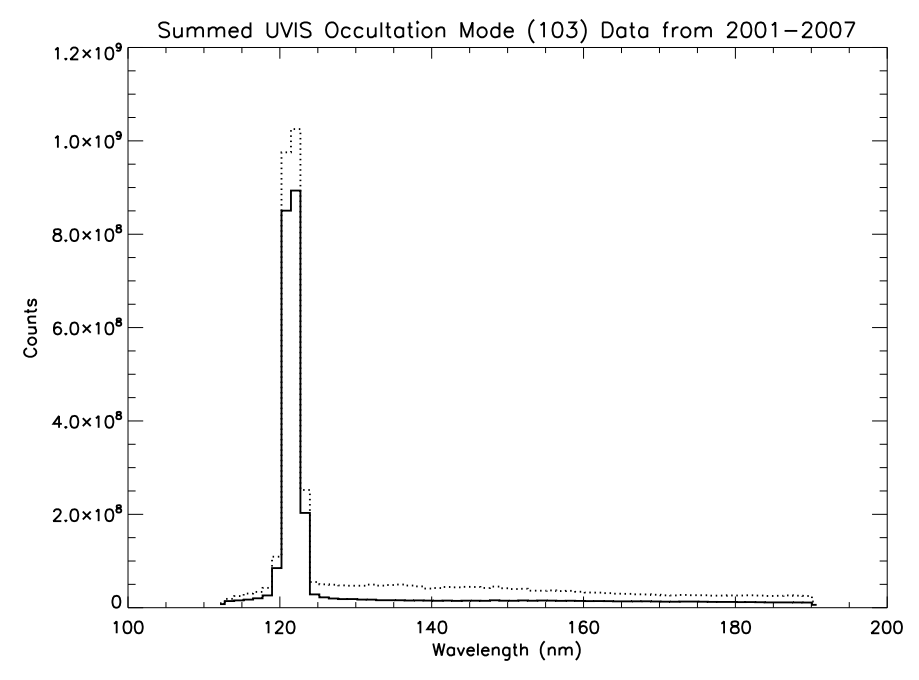

Fig. 3. Summed UVIS interplanetary Lyman- $\alpha$ spectrum from 2001-2007 obtained in the widest-slit mode (occultation mode, also called mode 103). The hydrogen line is spectrally unresolved. The upper curve in the figure contains Lyman- $\alpha$ and starlight at longer wavelengths. Obvious stars have been filtered to create the lower curve and the following Cassini data figures.

model of the heliospheric interface with interstellar parameters described in Table 2 ("Model 2"). The hydrogen bulk velocity used is $20 \mathrm{~km} \mathrm{~s}^{-1}$ (Clarke et al. 1998), with a temperature of $12000 \mathrm{~K}$ based on estimates from SOHO SWAN H absorption cell data (Costa et al. 1999).

The degree of damping cannot be estimated without a reliable model for solar activity. Measured solar Lyman- $\alpha$ values were provided by Tom Woods (Woods et al. 2000) based on measurements from Earth orbit by SME (Solar Mesosphere Explorer), UARS SOLSTICE, SORCE (Solar Radiation and Climate Experiment) SOLSTICE (McClintock et al. 2005), and TIMED SEE (Woods et al. 2005). These are line-integrated measurements of the output of the entire Earth-facing hemisphere of the Sun. Next, the amount of line-center Lyman- $\alpha$ radiation available to excite interplanetary gas is estimated based on work by Emerich et al. (2005) using the SOHO SUMER (Solar Heliospheric Observatory Solar Ultraviolet Measurements of Emitted Radiation) instrument. They found the relationship between line-center and line-integrated flux to be:

$f=0.64 F^{1.21} \pm 0.08$

where $f$ is the line-center solar spectral Lyman- $\alpha$ photon irradiance, expressed in units of $10^{12} \mathrm{~s}^{-1} \mathrm{~cm}^{-2} \mathrm{~nm}^{-1}$ and $F$ is the total Lyman- $\alpha$ photon irradiance, expressed in units of $10^{11} \mathrm{~s}^{-1} \mathrm{~cm}^{-2}$. This expression has the effect of varying the ratio of line-center to line-integrated flux from $\sim 0.85$ at solar minimum to $\sim 0.95$ at solar maximum. This expression also affects the derived solar Lyman- $\alpha$ radiation pressure used in determining the hydrogen atom trajectories in the hot model.

Our model also includes the time-dependence of two key loss processes for neutral hydrogen. The largest loss process is charge-exchange with solar wind protons, producing fast hydrogen atoms unable to scatter the solar line because of their large Doppler shifts. Solar wind mass flux variability (Pryor et al. 2003) is included in the model using the OMNI database produced by the NSSDC. The time-dependence of a second major loss process, EUV photoionization of neutral hydrogen, is included using photoionization estimates taken from the Solar2000 Model (Tobiska et al. 2000, 2006). 

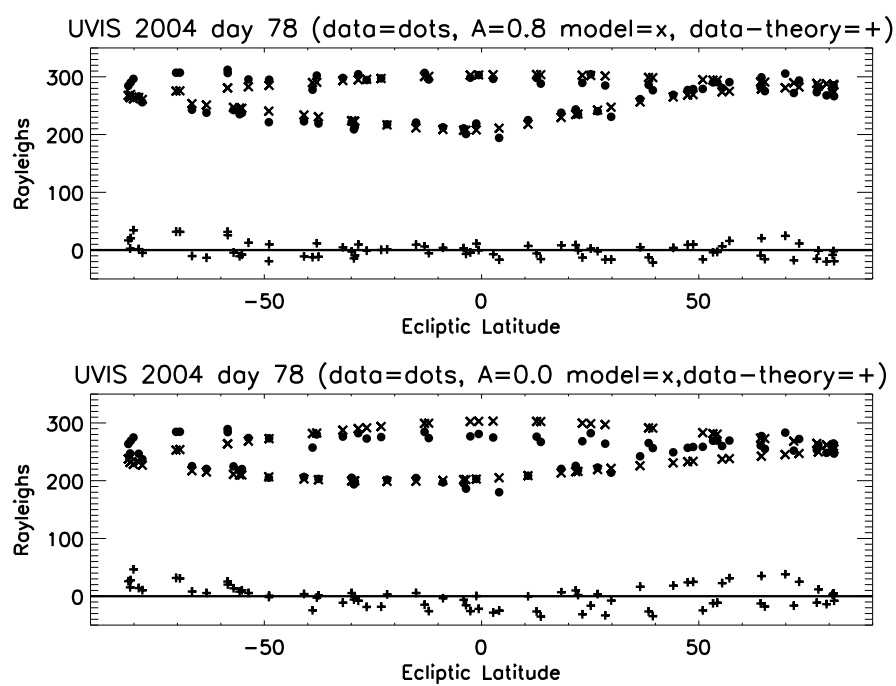

Fig. 4. Cassini UVIS Lyman- $\alpha$ data from spacecraft rolls on 2004 day 78 is plotted against ecliptic latitude and compared to two models: the top panel shows a model with a charge-exchange lifetime parameter of $A=0.8$, and the bottom panel shows a model with $A=0.0$. The $A=0$ case which corresponds to a spherically symmetric solar wind mass flux provides a worse fit to the data than the $A=0.8$ case. The upwind direction is near 250 degrees longitude and contains the maximum emission region. The brighter points on the figure (upper arcs) were obtained in the upwind hemisphere near ecliptic longitude 188 degrees, while the lower arcs were obtained in the downwind hemisphere near ecliptic longitude 8 degrees.

The amount of line-center Lyman- $\alpha$ seen at each longitude from Earth is used to infer the Lyman- $\alpha$ signal seen from the spacecraft in a line-of-sight integration through source regions at a variety of solar longitudes. The hydrogen density model used to do this is a modified hot model based on the work of Thomas (1978), and includes a variety of modifications discussed primarily in Pryor et al. (1992) to cope with latitude and longitude effects in Lyman- $\alpha$. We did not use our He $1083 \mathrm{~nm}$ technique (Pryor et al. 1996) for modeling Lyman- $\alpha$ data detailed variations in latitude as well as longitude because the National Solar Observatory He $1083 \mathrm{~nm}$ data sets are in transition to new instrumentation at this time.

The model needs to be slightly tuned to fit the spatial variations across the sky. The major remaining free parameter to do this is the " $A$ " parameter that controls the solar latitude dependence of the charge-exchange lifetime of neutral hydrogen $\tau_{\mathrm{sw}}$. The formula is (Witt et al. 1979):

$\tau_{\text {sw }}($ latitude $)=\tau_{\text {sw }}($ equator $) /\left(1-A \sin ^{2}(\right.$ latitude $\left.)\right)$.

Lyman- $\alpha$ data from UVIS obtained during individual Cassini spacecraft rolls in 2004 (near solar minimum) indicate that a model $A$ parameter value of 0.8 fits the Cassini data better than an $A$ parameter value of 0.0 (Fig. 4). $A=0$ is appropriate for a spherically symmetric solar wind; $A>0$ is more appropriate for enhanced solar wind mass flux near the solar equator.

When applied to the Cassini UVIS time-series Lyman- $\alpha$ upwind data obtained from downwind near Saturn's orbit in 2003-2004, the preferred model (using $A=0.8$ ) has time variations that track the Lyman- $\alpha$ data variations. Data were obtained with the UVIS FUV detector in 24-25 s integration intervals. Data were selected when the spacecraft was pointing in the upwind hemisphere within 30 degrees of the ecliptic plane. Periods with obvious stars contaminating the data

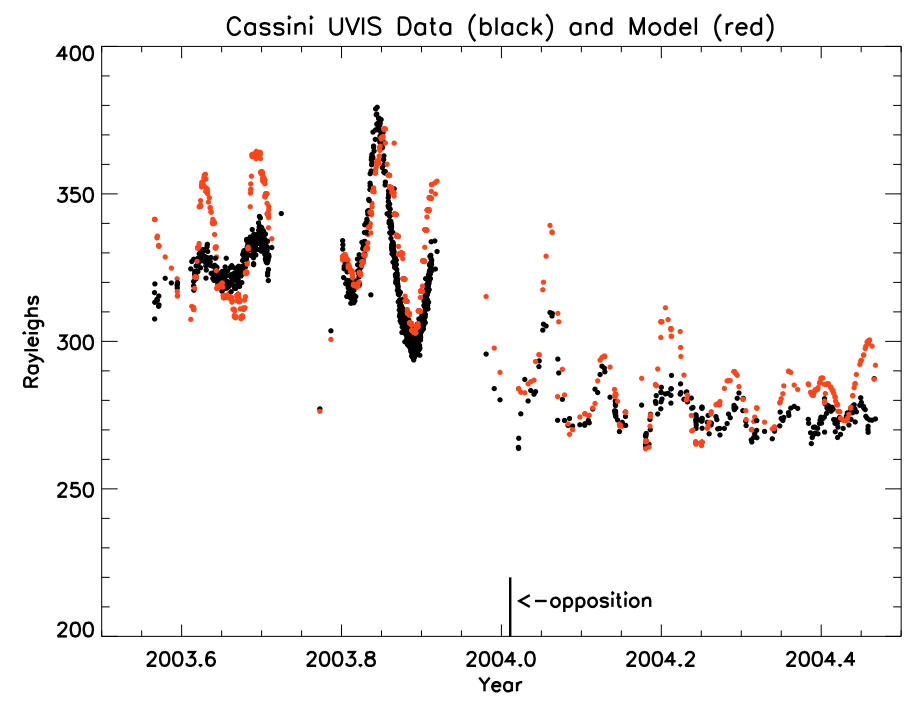

Fig. 5. UVIS data modulations observed in the upwind hemisphere from downwind on Saturn approach in the period 2003.5-2004.5 are generally in good agreement with the optically thin model using $A=0.8$, with solar rotations near Cassini opposition in early 2004 modeled better than the others.

were removed by comparing individual spectra to an uncontaminated Lyman- $\alpha$ spectra using a least-squares fit test. Figure 5 shows the rough agreement between a model and data obtained in 2 different instrument configurations: configuration 103 for occultation slit mode $(8 \mathrm{mrad} \times 60 \mathrm{mrad})$, and configuration 104 for low-resolution slit mode $(1.5 \mathrm{mrad} \times 60 \mathrm{mrad})$. Data (and model) were binned in time (by 80 24-s integrations for occultation mode, and by 192 24-s samples for low-resolution mode) to improve the signal-to-noise ratio. The results are largely independent of slit width, as they should be for a diffuse source with well-understood detector backgrounds. During some solar rotations the model modulations are the same size as the data modulations within a few percent. The agreement is best near opposition (early January, 2004) when the Sun, Earth, and Cassini are aligned. This is related to better knowledge of the solar Lyman- $\alpha$ flux on the side of the Sun facing Earth. We interpret the agreement between data and model near opposition to mean that for Cassini, the hydrogen column between the Sun, the relatively near-Sun scattering points that dominate the observed intensity, and the observer remains optically thin.

When the same single-scattering model is applied to the Voyager 1 data from 2003-2004 (Figs. 6, 7), damping is seen: the periodic waves in the data, while statistically significant, are much smaller than the waves in the optically thin model. We estimate the damping factor from the data and model comparison as follows. First, the average value of the data was scaled to the average value of the model, creating an empirical calibration factor. Next, a least-squares fit of a line to an 81-day running smooth of the model is subtracted from the model and the data to obtain detrended data and model, leaving the waves but no mean offset from 0 . Then, a least-squares fit of the detrended data to the detrended model was used to find the damping factor of $0.21 \pm$ 0.02 . That is, the 27 -day wave amplitude is about 5 times smaller in the data than in the optically thin model. The resulting fit of a "damped" model with waves reduced in amplitude by a factor of 0.21 to the original data is plotted in Fig. 7. The conclusion is that as Voyager 1 has traveled from $56 \mathrm{AU}$ to $88-92 \mathrm{AU}$, the damping factor has dropped from $\sim 0.4$ (Quemerais et al. 1996) to the new value of $\sim 0.2$, a trend anticipated by those authors. 


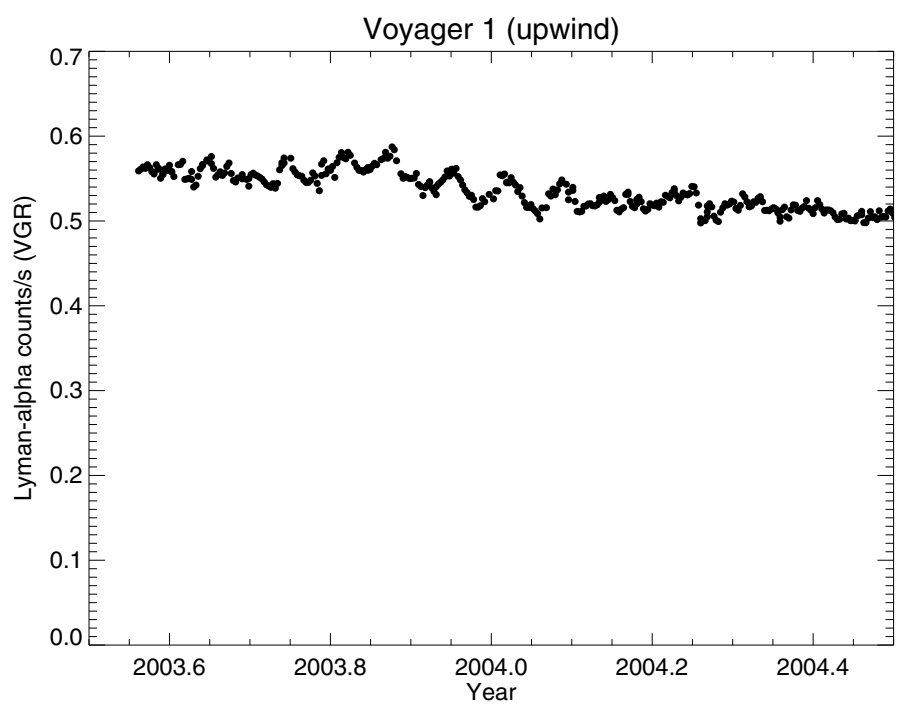

Fig. 6. Voyager 1 Lyman- $\alpha$ daily average count rates for the same period were obtained with the spacecraft upwind (88-92 AU from the Sun, at ecliptic longitude 251-252 degrees, ecliptic latitude 34 degrees) looking generally upwind (ecliptic longitude 263 degrees, ecliptic latitude 20 degrees). A 3-day running smooth has been applied to the data. Estimated statistical errors are 1-2 percent of the daily average values. Statistically significant modulations due to the Sun's rotation are observed in this period.

Interpretation of the damping factor in terms of hydrogen densities requires additional modeling with Monte Carlo techniques, to be discussed below.

\section{Multiple scattering models}

A preliminary approach to studying the modulation is to compare the new results with published curves in Quemerais et al. (1996). In this case examination of Fig. 6 in that paper indicates their models never covered damping as extreme as seen in the newer Voyager data. Nevertheless, it appears by extrapolation that wave damping would reach a factor of 5 near $120 \mathrm{AU}$ in their calculation, for a termination shock hydrogen density of $0.15 \mathrm{~cm}^{-3}$. To move this degree of damping inwards to $90 \mathrm{AU}$ where the new Voyager 1 data were acquired requires INCREASING the density by a factor of $4 / 3$, or creating a termination shock value of $0.20 \mathrm{~cm}^{-3}$. Three possible problems with this approach include:

1) their hydrogen "hot model" uses a constant hydrogen density in the outer heliosphere and neglects the outer heliospheric hydrogen wall that may begin to increase the damping in the most recent data;

2) extrapolation here is difficult to do accurately;

3) the model was run for a heliospheric $\mathrm{H}$ temperature near the termination shock of $8000 \mathrm{~K}$; more recent work with the SOHO (Solar and Heliospheric Observatory) SWAN (Solar Wind Anisotropy Experiment) absorption cell suggests a better temperature value to use in a hot model is $11500 \pm 1500 \mathrm{~K}$ (Costa et al. 1999). This change should affect the estimate as follows: the expression for line center optical depth is (Hall 1992) proportional to density divided by the square root of temperature. Thus raising the temperature from 8000 to $11500 \mathrm{~K}$ reduces the line-center optical depth at a given distance from the Sun by a factor of the square root of $(8000 / 11500)=0.83$. Thus Quemerais et al. (1996) overestimated optical depths by 17 percent for a given density

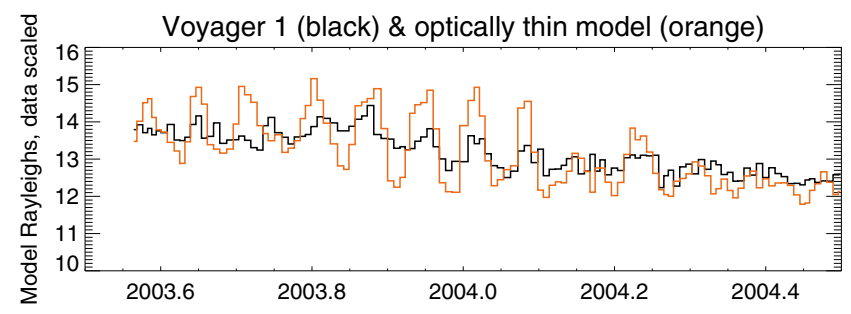

Voyager 1 (black), thin model (orange) amp. modulated by $0.21, r=0.44$

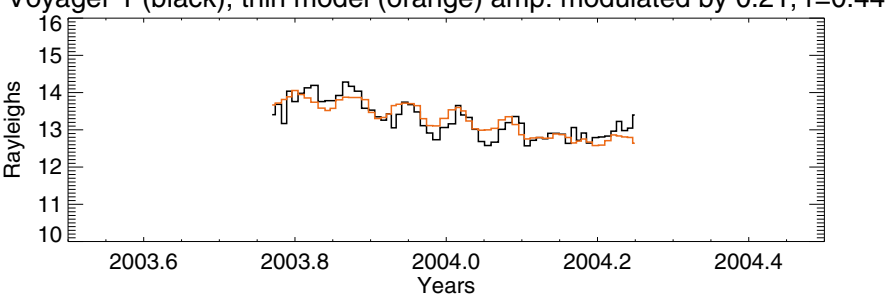

Fig. 7. Top panel: comparing the Voyager 1 data from this period to the optically thin model shows many of the same features are present in both time-series, but with greatly reduced amplitude in the data. This indicates that multiple scattering is important at this large distance from the Sun. Bottom panel: improved agreement between the model and the Voyager 1 data from late 2003 to early 2004 is obtained in this case by reducing the amplitude of the 27-day waves in the optically thin model by a damping factor of 0.21 . The interval plotted is shorter to avoid edge effects from the 81-day smoothing used to detrend the data.

and distance, raising the estimated termination shock density derived from the damping data presented here by 17 percent from 0.2 to $0.23 \mathrm{~cm}^{-3}$ for a hot model (that neglects the hydrogen wall). This rather large density estimate may indicate that the spacecraft observations are beginning to sample the larger hydrogen densities beyond the termination shock in the hydrogen wall.

A second, probably better approach to the new data is to use a Monte Carlo radiative transfer model (Gangopadhyay et al. 2002, 2006) for wave damping by artificially placing a bright spot 10 degrees in radius on the Sun alternately on the upwind axis and the downwind axis. This radiative transfer model uses the distribution of hydrogen obtained in the frame of a stationary Baranov-Malama model of the heliospheric interface. Basic results of the model are reported in recent reviews by Baranov \& Izmodenov (2006) and Izmodenov \& Baranov (2007). This spot or "active region" is given an enhanced output in accordance with the "contrast factor" of 4.6 compared to non-active regions presented for Lyman- $\alpha$ active regions in Cook et al. (1981). The upwind maximum heliospheric Lyman- $\alpha$ intensity is obtained for the spot upwind, while the upwind minimum is obtained for the spot downwind. These two extreme cases correspond to the intensity maxima and minima seen in the Voyager data. The damping of this modulation with distance is directly comparable to the damping seen in the Voyager upwind data. We performed radiative transfer calculations by using hydrogen distributions (Fig. 8) obtained in the frame of a modified Baranov-Malama model that includes influences of interstellar helium ions and solar wind $\alpha$ particles (Izmodenov et al. 2003) with two different sets of interstellar proton and neutral hydrogen number densities (see Table 1). Other interstellar and solar boundary parameters remain the same for the two sets of calculations. Local interstellar velocity and temperature were assumed as $26.4 \mathrm{~km} \mathrm{~s}^{-1}$ and $6500 \mathrm{~K}$ that is closer to the values derived from measurements of interstellar helium inside the heliosphere (Witte et al. 1996; Witte 2004; Möbius et al. 2004). Solar wind number 
Table 1. Two-shock heliospheric models compared to Voyager data.

\begin{tabular}{lcccccc}
\hline \hline Model & $\begin{array}{c}\text { Proton } \\
\text { density at } \\
\text { the outer } \\
\text { boundary } \\
\left(\mathrm{cm}^{-3}\right)\end{array}$ & $\begin{array}{c}\text { H neutral } \\
\text { density at } \\
\text { the outer } \\
\text { boundary } \\
\left(\mathrm{cm}^{-3}\right)\end{array}$ & $\begin{array}{c}\text { H neutral } \\
\text { density at } \\
\text { termination } \\
\text { shock } \\
\left(\mathrm{cm}^{-3}\right)\end{array}$ & $\begin{array}{c}\text { H neutral } \\
\text { density at } \\
90 \mathrm{AU} \\
\left(\mathrm{cm}^{-3}\right)\end{array}$ & $\begin{array}{c}\text { Termination } \\
\text { shock } \\
\text { distance } \\
(\mathrm{AU})\end{array}$ & $\begin{array}{c}\text { Damping } \\
\text { factor } D \\
\text { at 90 AU }\end{array}$ \\
\hline Model 1 & 0.06 & 0.18 & 0.095 & 0.092 & 97 & $0.27 \pm 0.07$ \\
$\begin{array}{l}\text { Model 2 } \\
\text { Voyager } \\
\text { 1 data }\end{array}$ & 0.05 & 0.15 & 0.085 & 0.082 & 106 & $0.24 \pm 0.07$ \\
\hline
\end{tabular}

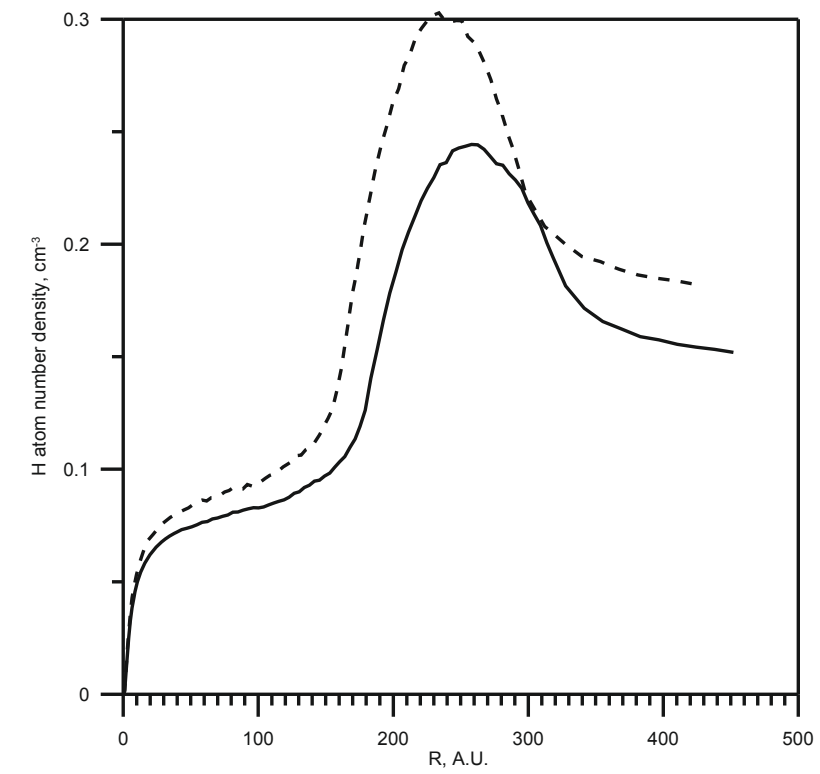

Fig. 8. The radial hydrogen density profile in the upwind direction is shown for the two models described in Table 1. Model 1 is the upper curve and has a termination shock distance of $97 \mathrm{AU}$ and Model 2 is the lower curve with a termination shock distance of 106 AU. A hydrogen wall is present (local maximum) between 200 and $300 \mathrm{AU}$ in both models. The Voyager data modeled here involve upwind lines-of-sight that sample a region of increasing density with distance.

density and velocity at the Earth's orbit were assumed as $7.39 \mathrm{~cm}^{-3}$ and $432 \mathrm{~km} \mathrm{~s}^{-1}$. For each model over 10 million photons were launched in the Monte Carlo simulation and propagated through the heliosphere to examine the damping of the modulation with distance. The final run of the completed radiative transfer model took 2 months of computer time. The error estimates in Table 1 reflect the observed scatter in the Monte Carlo results.

Model modulation $M$ is here defined as:

$M=\left(I_{\text {upwind } \& \text { spot upwind }}-I_{\text {upwind } \& \text { spot downwind }}\right) / I_{\text {upwind } \& \text { spot upwind }}$

where $I_{\text {upwind \& spot upwind }}$ is the upwind intensity when the spot is upwind and $I_{\text {upwind \& spot downwind }}$ is the upwind intensity when the spot is downwind. The modulation in the upwind direction is somewhat larger in the denser Model 1 (Fig. 9), because photons from the downwind spot have a harder time scattering all the way around the Sun to upwind in the denser hydrogen cloud. We tested this interpretation by also running a case for each model with no spot at all, and found that adding a downwind spot contributed about 10 percent of the upwind intensity in the less dense Model 2 and contributed a negligible amount to the upwind intensity in the somewhat denser Model 1.
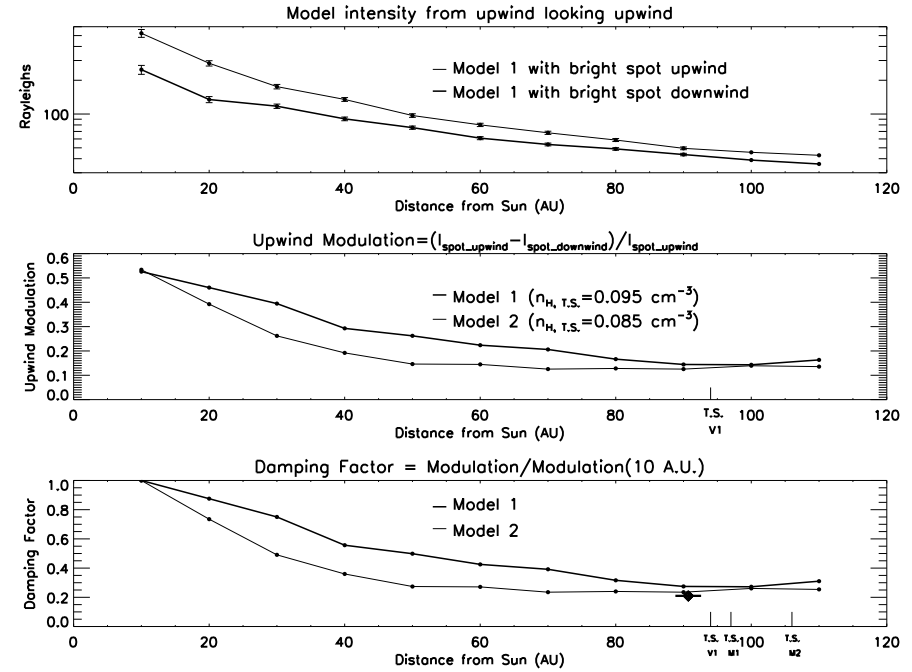

Fig. 9. Top panel: Monte Carlo simulations of the Lyman- $\alpha$ intensity upwind viewed from the upwind axis as a function of heliocentric distance using Model 1 for 2 extreme cases: a bright spot on the Sun on the upwind axis, and a bright spot on the Sun on the downwind axis. Calculations were performed at 10 AU steps. 1-sigma statistical error bars are shown. Middle panel: the expected "27-day" upwind modulation in the Lyman- $\alpha$ brightness as seen from the upwind axis as a function of heliocentric distance is shown for Models 1 and 2, computed from the 2 extreme cases in the top panel. Displayed values in the lower 2 panels are a 3-point running average to minimize statistical fluctuations. Bottom panel: the damping factor upwind (modulation divided by the modulation at $10 \mathrm{AU}$ from the Sun) as seen from the upwind axis as a function of heliocentric distance. The measured damping for the Voyager 1 data is also indicated (heavy plus sign near 90 AU from the Sun). Termination shock (T.S.) locations for Voyager 1, Models 1 and 2 are also shown.

Model damping, $D$, is defined as

$D=M($ distance $) / M($ at $10 \mathrm{AU})$

that is, the modulation at a given distance divided by the modulation at $10 \mathrm{AU}$ from the Sun. $10 \mathrm{AU}$ is used as the reference because it was the first grid point in the calculation and should be undamped or almost undamped. The modulation damping near 90 AU in both Models 1 and 2 (Fig. 9) is similar to the modulation damping found in the Voyager data near 90 AU. Note that the statistical errors in the Monte Carlo model modulation and damping are sizeable (20-30 percent) because they involve differences of similar quantities. Therefore we should not over-interpret these calculations, but it appears that densities in these models are reasonably close to the truth in the sense that the damping and associated error-bars for the Models 1 and 2 $(0.27 \pm 0.07$ and $0.24 \pm 0.07$ respectively) overlap the error-bars 
Table 2. Estimates of neutral H density (near termination shock TS) from Lyman- $\alpha$ wave damping with distance.

\begin{tabular}{ccccc}
\hline \hline Reference & Data source & $\begin{array}{c}\text { Observation } \\
\text { Year }\end{array}$ & $\begin{array}{c}\text { Distance to Sun } \\
\text { (AU) }\end{array}$ & $\begin{array}{c}\text { H density at TS } \\
\left(\mathrm{cm}^{-3}\right)\end{array}$ \\
\hline Shemansky et al. (1984) & Pioneer 10 & 1982 & 30 & $0.11-0.12$ \\
Shemansky et al. (1984) & Voyager 2 & 1982 & 12 & $0.16-0.17$ \\
Quemerais et al. (1996) & Voyager 1, 2 & $1981-1993$ & up to 56, 44 & $0.15 \pm 0.10$ \\
This paper & Cassini UVIS, & $2003-2004$ & 10,90 & $\sim 0.085-0.095$ \\
Pryor et al. (2008) & Voyager & & & \\
\hline
\end{tabular}

on the observed damping in the Voyager data $(0.21 \pm 0.02)$. A possible problem with this approach is that the degree of damping may be somewhat sensitive to the assumed solar brightness distribution (see Quemerais et al. 1996). The two models shown here are not a full parametric study, and additional model constraints are needed to arrive at a clear picture.

\section{Conclusions}

While the damping factor compared to the well-established hot models is a fairly robust result, derivation of the hydrogen density is more model-dependent. For example, in a strictly singlescattering model, the waves should be undamped with distance.

Figure 6 clearly rules out models that do not contain significant amounts of multiple scattering in forming the observed Lyman- $\alpha$ signal at Voyager. This is an important result, as a research paper by Scherer (1996) and a review chapter by Scherer (2000) both discount the importance of Lyman- $\alpha$ multiple scattering in the heliosphere based on their radiative transfer calculations. Scherer \& Scherer (2001), also argued that the Pioneer 11 data out to $30 \mathrm{AU}$ demonstrate optically thin behavior, because they observe a constant $\mathrm{H} / \mathrm{He} \mathrm{UV}$ emission ratio from 1972-1984. This argument seems incomplete without consideration of the different solar cycle variations in the two solar lines, H Lyman- $\alpha 121.6 \mathrm{~nm}$ and He $58.4 \mathrm{~nm}$, that excite the interplanetary emissions. Their paper also shows the ratio is very different for Pioneer 10 and 11, perhaps indicating instrumental changes, probably in Pioneer 10. This discrepancy makes it difficult to draw firm conclusions from the long-term time-series data, although it would be a valuable project to see if existing $\mathrm{H}$ and $\mathrm{He}$ models can reproduce the observed trends from either spacecraft.

On the other hand, Keller \& Thomas (1979), Keller et al. (1981), Hall (1992), Hall et al. (1993), Quemerais \& Bertaux (1993), Quemerais et al. (1996), and Gangopadhyay et al. (2006) have all calculated a major role for multiple scattering in the outer heliosphere. Our observational results support this theoretical conclusion: "27-day" waves seen in the Voyager outer heliosphere data are damped compared to well-tested optically thin heliospheric hot models, but are more consistent with the expectations of multiple scattering calculations.

Derived density values are likely to remain controversial. Density values computed strictly based on UV calibrations have led to a wide variety of results, with a larger spread of results than is seen in our Table 2 (e.g., Ajello et al. 1987; Quemerais et al. 1994). The results presented here, based on the damping technique and the best available models for heliospheric hydrogen, are a step towards improved understanding of the hydrogen densities.

Figure 9 shows that the damping behavior with distance is somewhat different for the two different heliospheric models, suggesting that it will be valuable in the future to compute the damping factor in the Voyager data as a function of distance over the mission and use it to discriminate between different proposed heliospheric models. This will require extensive computation to further reduce the error bars on the damping seen in the Monte Carlo simulations.

Comparison with other hydrogen density determination techniques is the main point of this special section of the journal based on an ISSI team effort (Möbius et al. 2005). Measurements of $\mathrm{H}$ absorption in spectra of the closest stars indicate the average neutral $\mathrm{H}$ density along the line of sight to be close to $0.1 \mathrm{~cm}^{-3}$ (Fig. 14 in Wood et al. 2005). On the other hand, Slavin \& Frisch (2008) calculate that the neutral H density just outside the heliosphere in the Circum-Heliosphere Interstellar Cloud (CHIC) is higher, $0.19-0.20 \mathrm{~cm}^{-3}$, (with an estimated electron density $=0.05-0.08 \mathrm{~cm}^{-3}$ ) because we are in one of a cluster of local interstellar clouds. Their calculation is based on radiative transfer modeling of the sightline to the hot star Epsilon Canis Majoris, using helium, nitrogen and oxygen constraints. Heliospheric models then typically reduce these outer boundary condition hydrogen density values by a filtration factor due to charge-exchange during passage through about $200 \mathrm{AU}$ of heliosheath to obtain the interstellar hydrogen density at the termination shock. A suite of such models examined by Mueller et al. (2008) had neutral hydrogen filtration factors of 0.52-0.74. Models 1 and 2 compared to data here (Table 1) from Izmodenov used interstellar values of 0.18 and $0.15 \mathrm{~cm}^{-3}$ respectively for the neutral hydrogen density, with electron densities in 0.06 and $0.05 \mathrm{~cm}^{-3}$ in general agreement with the Slavin \& Frisch (2008) boundary condition, although Model 1 is in better agreement with their results. After filtration, the termination shock values for neutral hydrogen were 0.095 and $0.085 \mathrm{~cm}^{-3}$ respectively. The termination shock locations in the two models were at 97 and $106 \mathrm{AU}$, in reasonable agreement with the first reported termination shock crossing at 94 AU on 16 Dec. 2004 by Voyager 1 (Stone et al. 2005).

Our results for the $\mathrm{H}$ density can be compared with results from pickup proton density measurements that lead to neutral hydrogen density estimates near the termination shock (Gloeckler \& Geiss 2001; Izmodenov et al. 2003) with a most recent neutral hydrogen density estimate of $0.11 \pm 0.022 \mathrm{~cm}^{-3}$ at the termination shock (Bzowski et al. 2008) after filtration from local interstellar cloud values of neutral hydrogen density $0.195 \pm 0.02 \mathrm{~cm}^{-3}$, and a plasma proton density $0.04 \pm$ $0.02 \mathrm{~cm}^{-3}$. Another indicator of the $\mathrm{H}$ density is solar wind slowdown in the outer heliosphere due to mass loading by pickup hydrogen ions, leading to a hydrogen density estimate of $0.09 \pm$ $0.01 \mathrm{~cm}^{-3}$ at the termination shock (Richardson et al. 2008). Thus it appears that a consistent picture is slowly emerging of the heliospheric hydrogen densities near the termination shock based on these various techniques.

Acknowledgements. We acknowledge support from two ISSI team projects to evaluate interstellar $\mathrm{H}$ parameters. ISSI, the International Space Science Institute, is based in Bern, Switzerland. Wayne Pryor also acknowledges support from the NASA Heliospheric Missions Guest Investigator Program, the 
NASA JPL Cassini Project, Hampton University, Central Arizona College and the University of Colorado. It is a pleasure to acknowledge the computational support given by the University of Southern California High Performance Computing and Communication (HPCC) center. Pradip Gangopadhyay was supported in part by NASA grant NNG04GB80G. Eberhard Möbius gratefully acknowledges support for this study from NASA grant NNG06GD55G. V. Izmodenov was supported by RFBR grants 05-02-22000(PICS), 07-02-01101 and "Dynastia" Foundation. Yu. G. Malama was supported by RFBR grant 0602-7255. Tom Woods provided the composite Lyman-alpha data used in this analysis. We acknowledge helpful discussions with Priscilla Frisch on the heliospheric boundary conditions.

\section{References}

Adams, T. F., \& Frisch, P. C. 1977, ApJ, 212, 300

Ajello, J. M., Stewart, A. I., Thomas, G. E., \& Graps, A. 1987, ApJ, 317, 964

Baranov, V. B., \& Malama, Yu. G. 1993, J. Geophys. Res., 98, 15157

Baranov, V. B., \& Izmodenov, V. V. 2006, Fluid. Dyn., 41, 689

Baranov, V. B., Lebedev, M. G., \& Malama, Yu. G. 1991, ApJ, 375, 347

Bertaux, J. L., \& Blamont, J. E. 1971, A\&A, 11, 200

Broadfoot, A. L., Sandel, B. R., Shemansky, D. E., et al. 1977, Space Sci. Rev., 21,183

Bzowski, M., Möbius, E., Tarnopolski, S., Izmodenov, V., \& Gloeckler, G. 2008, A\&A, 491, 7

Carlson, R. W., \& Judge, D. L. 1974, J. Geophys. Res., 79, 3623

Clarke, J. T., Lallement, R., Bertaux, J.-L., et al. 1998, ApJ, 499, 482

Cook, J. W., Meier, R. R., Brueckner, G. E., \& Van Hoosier, M. E. 1981, A\&A, 97, 394

Costa, J., Lallement, R., Quemerais, E., et al. 1999, ApJ, 349, 660

Emerich, C., Lemaire, P., Vial, J.-C., et al. 2005, Icarus, 178, 429

Esposito, L. W., Barth, C. A., Colwell, J. E., et al. 2004, Space Sci. Rev., 115, 299

Gangopadhyay, P., Izmodenov, V., Gruntman, M., \& Judge, D. L. 2002, J. Geophys. Res., 107, A11, 1387

Gangopadhyay, P., Izmodenov, V. V., Gruntman, M., \& Judge, D. L. 2006, ApJ, 637, 786

Gloeckler, G., \& Geiss, J. 2001, in Solar and Galactic Composition: A Joint SOHO/ACE Workshop, ed. R. F. Wimmer-Schweingruber, AIP Conf. Proc., 598,281

Hall, D. T. 1992, Ultraviolet Resonance Scattering and the Structure of the Heliosphere, Ph.D. Thesis, Physics Dept. (Tucson: University of Arizona)

Hall, D. T., Shemansky, D. E., Judge, D. L., Gangopadhyay, P., \& Gruntman, M. A. 1993, J. Geophys. Res., 98, 15185

Izmodenov, V. V., \& Baranov, V. B. 2007, in The physics of the heliospheric boundaries, ed. V. V. Izmodenov, \& R. Kallenbach (Noordwijk, the Netherlands: ESA Publications), ISSI Sci. Rep. Ser., 5, 67

Izmodenov, V. V., Gruntman, M., \& Malama, Y. G. 2001, J. Geophys. Res., 106, 10681

Izmodenov, V., Malama, Y. G., Gloeckler, G., \& Geiss, J. 2003, ApJ, 594, L59

Judge, D. L., \& Carlson, R. W. 1974, Science, 183, 317
Keller, H. U., \& Thomas, G. E. 1979, A\&A, 80, 227

Keller, H. U., Richter, K., \& Thomas, G. E. 1981, A\&A, 102, 415

Kurt, V. G. 1967, Issledovaniya Kosmicheskogo Prostranstva, 6, 911, in NASA Translation, ST-OA-SP-10669, 1968

Linsky, J. L., \& Wood, B. E. 1996, ApJ, 463, 254

McClintock, W. E., Rottman, G. J., \& Woods, T. N. 2005, Sol. Phys., 230, 225

Möbius, E., Bzowski, M., Chalov, S., et al. 2004, A\&A, 426, 897

Möbius, E., Bzowski, M., Fahr, H.-J., et al. 2005, Proceedings of Solar Wind 11/SOHO 16, Connecting the Sun and Heliosphere Conference, ESA SP-592

Mueller, H.-R., Florinski, V., Heerikhuisen, J., et al. 2008, A\&A, 491, 43

Pryor, W. R., Ajello, J. M., Barth, C. A., et al. 1992, ApJ, 394, 363

Pryor, W. R., Barth, C. A., Hord, C. W., et al. 1996, Geophys. Res. Lett., 23, 1893

Pryor, W. R., Ajello, J. M., McComas, D. J., Witte, M., \& Tobiska, W. K. 2003, J. Geophys. Res., 108, 8034

Quemerais, E., \& Bertaux, J.-L. 1993, A\&A, 277, 283

Quemerais, E., Bertaux, J.-L., Sandel, B. R., \& Lallement, R. 1994, A\&A, 290, 941

Quemerais, E., Sandel, B. R., \& de Toma, G. 1996, ApJ, 463, 349

Richardson, J. D., Liu, Y., Wang, C., \& McComas, D. J. 2008, A\&A, 491, 1

Scherer, H. 1996, A\&A, 309, 957

Scherer, H. 2000, in The Outer Heliosphere: Beyond the Planets, ed. K. Scherer, H. Fichtner, \& E. Marsch, 91

Scherer, H., \& Scherer, K. 2001, in The Outer Heliosphere: the Next Frontiers, proceedings of COSPAR colloquium in Potsdam, Germany, 24-28 July, 2000, ed. K. Scherer, H. Fichtner, H. Fahr, \& E. Marsch (Amsterdam: Pergamon Press)

Shemansky, D. E., Judge, D. L., \& Jesson, J. M. 1984, in Local Interstellar Medium, ed. F. C. Bruhweiler, Y. Kondo, \& B. D. Savage (NASA CP-2345), IAU Colloq., 81, 24

Slavin, J. D., \& Frisch, P. C. 2008, A\&A, 491, 53

Stone, E., Cummings, A. C., McDonald, F. B., et al. 2005, Science, 309, 2017

Thomas, G. E. 1978, Ann. Rev. Earth \& Planet. Sci., 6, 173

Thomas, G. E., \& Krassa, R. F. 1971, A\&A, 11, 218

Tobiska, W. K., \& Bouwer, S. D. 2006, Adv. Space Res., 37, 347

Tobiska, W. K., Woods, T., Eparvier, F., et al. 2000, J. Atm. \& Sol.-Terr. Phys., 62,1233

Witt, N., Ajello, J. M., \& Blum, P. W. 1979, A\&A, 73, 272

Witte, M. 2004, A\&A, 426, 835

Witte, M. 2007, unpublished memo

Witte, M., Banaszkiewicz, M., \& Rosenbauer, H. 1996, Space Sci. Rev., 78, 289

Wood, B. E., Redfield, S., Linsky, J. L., Muller, H.-R., \& Zank, G. P. 2005, ApJS, 159,118

Wood, B. E., Izmodenov, V. V., Linsky, J. L., \& Alexashov, D. 2007a, ApJ, 659, 1784

Wood, B. E., Izmodenov, V. V., Linsky, J. L., \& Malama, Y. G. 2007b, ApJ, 657, 609

Woods, T. N., Tobiska, W. K., Rottman, G. J., \& Worden, J. R. 2000, J. Geophys. Res., 105, 27195

Woods, T. N., Eparvier, F. G., Bailey, S. M., et al. 2005, J. Geophys. Res., 110, A01312 